\title{
Archéopages
}

Archéopages

Archéologie et société

\section{À la recherche des espaces de stabulation. Étude pluridisciplinaire d'habitats du Néolithique au Moyen Âge dans le nord de la France}

Frédéric Broes, Viviane Clavel, Wim De Clercq, Kai Fechner, Vanessa Rouppert et Jan Vanmoerkerke

\section{(2) OpenEdition}

Journals

Édition électronique

URL : https://journals.openedition.org/archeopages/257

DOI : $10.4000 /$ archeopages.257

ISSN : 2269-9872

Éditeur

INRAP - Institut national de recherches archéologiques préventives

Édition imprimée

Date de publication : 1 mai 2013

Pagination : 6-16

ISSN : $1622-8545$

\section{Référence électronique}

Frédéric Broes, Viviane Clavel, Wim De Clercq, Kai Fechner, Vanessa Rouppert et Jan Vanmoerkerke, «À la recherche des espaces de stabulation. Étude pluridisciplinaire d'habitats du Néolithique au Moyen Âge dans le nord de la France », Archéopages [En ligne], 35 | 10/2012, mis en ligne le 01 octobre 2014, consulté le 21 janvier 2022. URL : http://journals.openedition.org/archeopages/257 ; DOI : https://doi.org/10.4000/archeopages.257 


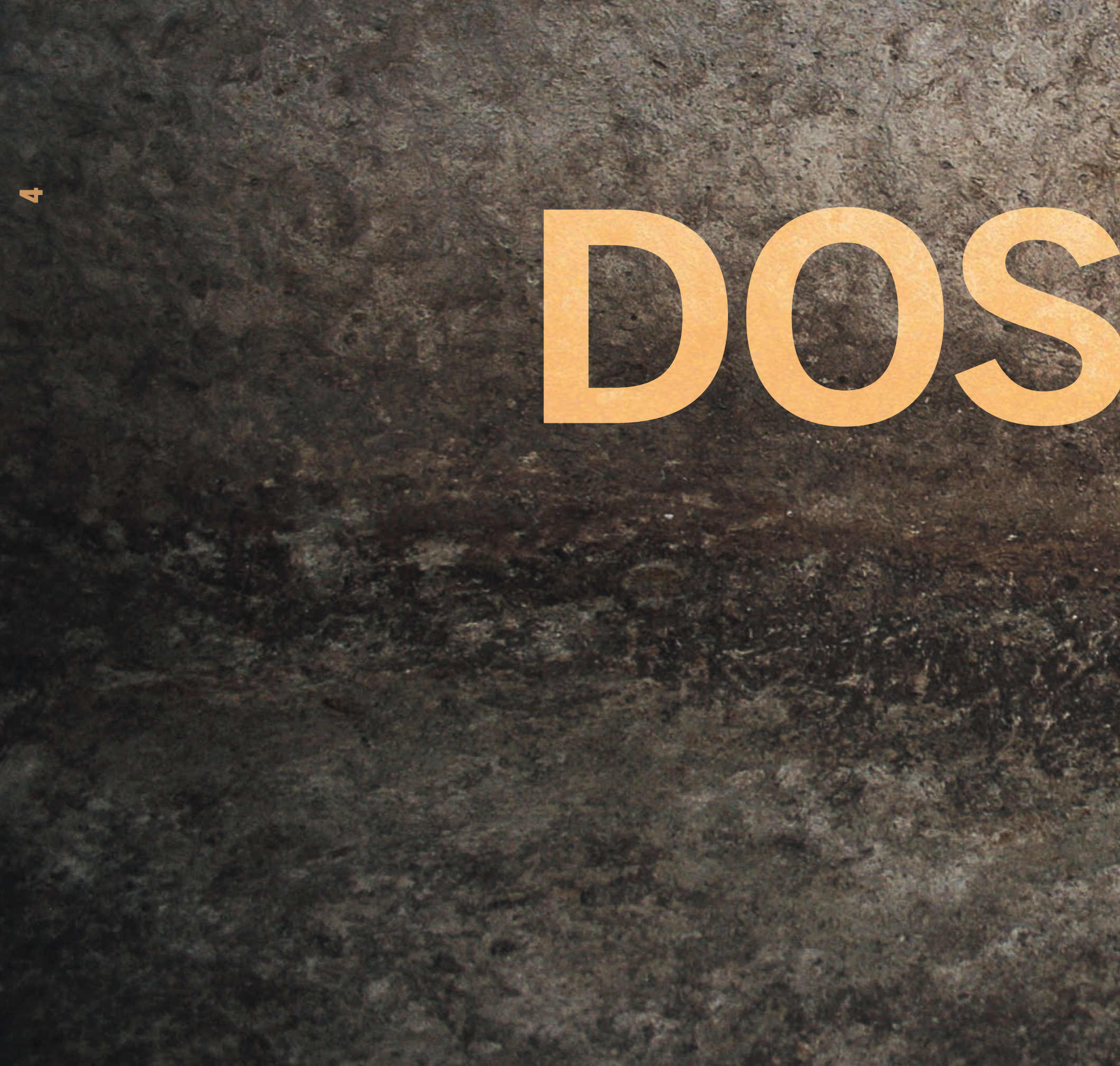




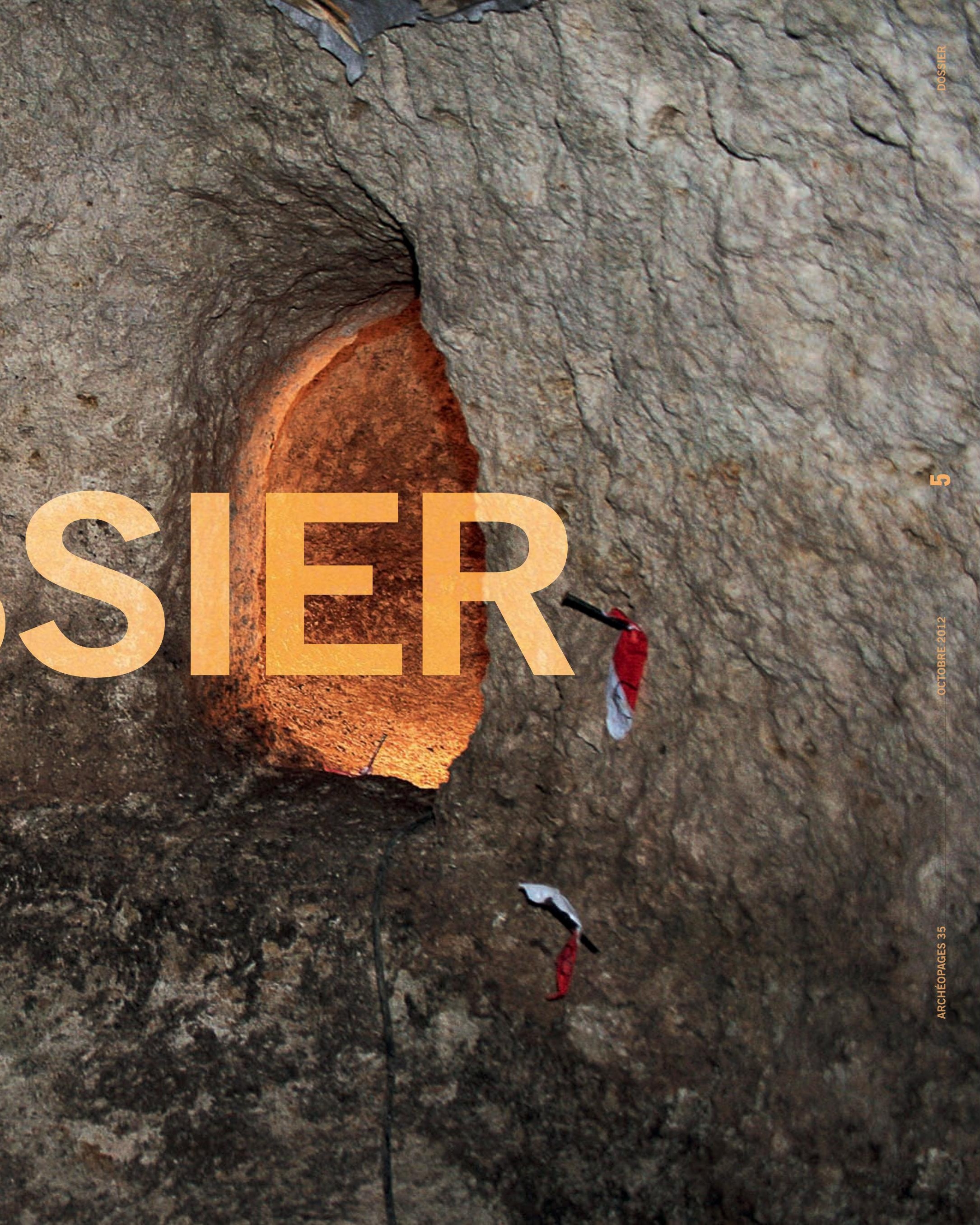




\title{
À la recherche des espaces
}

de stabulation

Étude pluridisciplinaire d'habitats du Néolithique au Moyen Âge dans le nord de la France

\author{
Frédéric Broes Inrap \\ Viviane Clavel Inrap \\ Wim De Clercq université de Gand \\ Kai Fechner Inrap, UMR 7356 LIVE \\ Vanessa Rouppert Inrap \\ Jan Vanmoerkerke ministere de la culture
}

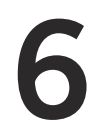

1. Avec la contribution de Cécilia Cammas,

Inrap/UMR 5140 DMOS Yves Créteur, Inrap,

Guillaume Hulin, Inrap/

UMR 7617 Sisyphe, Ivan

Praud, Inrap/UMR 8215

Trajectoires, Pascal

Verdin, Inrap/UMR 6130

CEPAM; Perrine

Gambier, CRAVO ;

Véronique Zech-Matterne,

CNRS/UMR 7209

Archéozoologie-

Archéobotanique.
Les indices découverts lors de fouilles de constructions anciennes permettent d'étayer l'hypothèse de leur fonction (espace artisanal, espace domestique, maison-étable, bâtiment d'élevage,...) : il s'agit d'éléments architecturaux (murs, sablières, trous de poteaux) et de leur disposition dans l'espace (partitions internes, relations entre structures); de la répartition des artefacts découverts dans les structures du bâtiment ou situés dans l'environnement proche, tels que les restes céramiques, lithiques ou métalliques ; et enfin, de la distribution spatiale des écofacts tels que les restes carpologiques (graines, reste de fourrage...), anthracologiques (charbons de bois) ou osseux et des modifications chimiques de l'encaissant $i n$ situ. Ce texte a pour objet de souligner la pertinence d'intégrer les résultats obtenus par les études environnementales aux indices visant à déterminer la fonction des bâtiments anciens excavés $\mathbf{1}$.

\section{État de la recherche typofonctionnelle} des bâtiments en archéologie

Depuis les travaux fondateurs de Van Giffen entre les deux guerres, puis de Zimmermann et de ses collègues à partir des années 1970 (Zimmerman, 1999), la recherche sur la stabulation animale et notamment sur les maisons-étables est bien ancrée en Europe nord-occidentale. Il s'agit d'une analyse fonctionnelle de tous les bâtiments, grands comme petits, avec une approche régressive : à partir de constructions dont la fonction est connue par d'autres arguments (stalles, restes de fourrage, de fumier...) [ill. 1], on essaie de remonter dans le temps, en suivant les moindres changements architecturaux et toutes les autres composantes de la société agricole. Ces modifications, parfois minimes, souvent fondamentales, sont également comparées entre régions voisines afin d'expliquer certaines variations géographiques architecturales par des différences fonctionnelles.

Ainsi, dans les Flandres, aux Pays-Bas, en Allemagne du Nord et dans le sud de la Scandinavie, grâce à un corpus de milliers de maisons, une tradition de maisons-étables est avérée du Bronze moyen au premier âge du Fer. Ailleurs, et pour des périodes plus anciennes, la situation est particulièrement complexe, mais ce type d'approche permet d'identifier d'éventuelles étables en Europe occidentale et centrale dès le Bronze ancien. Du Néolithique final au Néolithique moyen, les corpus sont insuffisants pour cette méthode, mais certaines caractéristiques de la stabulation existent dans une partie des bâtiments. Pour le Néolithique ancien, la multitude des plans connus, et leur standardisation combinée à la faiblesse des caractéristiques architecturales, ne plaident pas en faveur de la stabulation.

Pour la Protohistoire récente et pour les époques romaines et médiévales (d'environ 50 avant notre ère à 1200), l'interprétation typofonctionnelle de l'architecture en bois se fonde surtout sur les traces de stabulation, notamment sur l'emplacement des poteaux dans les maisons, ainsi que sur la présence, dans une partie de la maison, d'une légère excavation, appelée «potstal» en néerlandais [ill. 2]. Le modèle architectural de la maison-étable (la Wohnsthallhaus de la littérature allemande) a été particulièrement bien documenté sur les sols moins aptes à l'agriculture de surplus mais plus favorables à une économie agraire mixte ou surtout orientée vers l'élevage (Roymans, 1999).

Dès les années 1930, des maisons-étables ont été mises au jour : des chercheurs allemands et hollandais travaillant sur des sites côtiers stratifiés et sur des plateformes artificielles d'habitat («terpen») du nord des Pays-Bas et du nord-ouest de l'Allemagne ont mis au jour des maisons en bois 


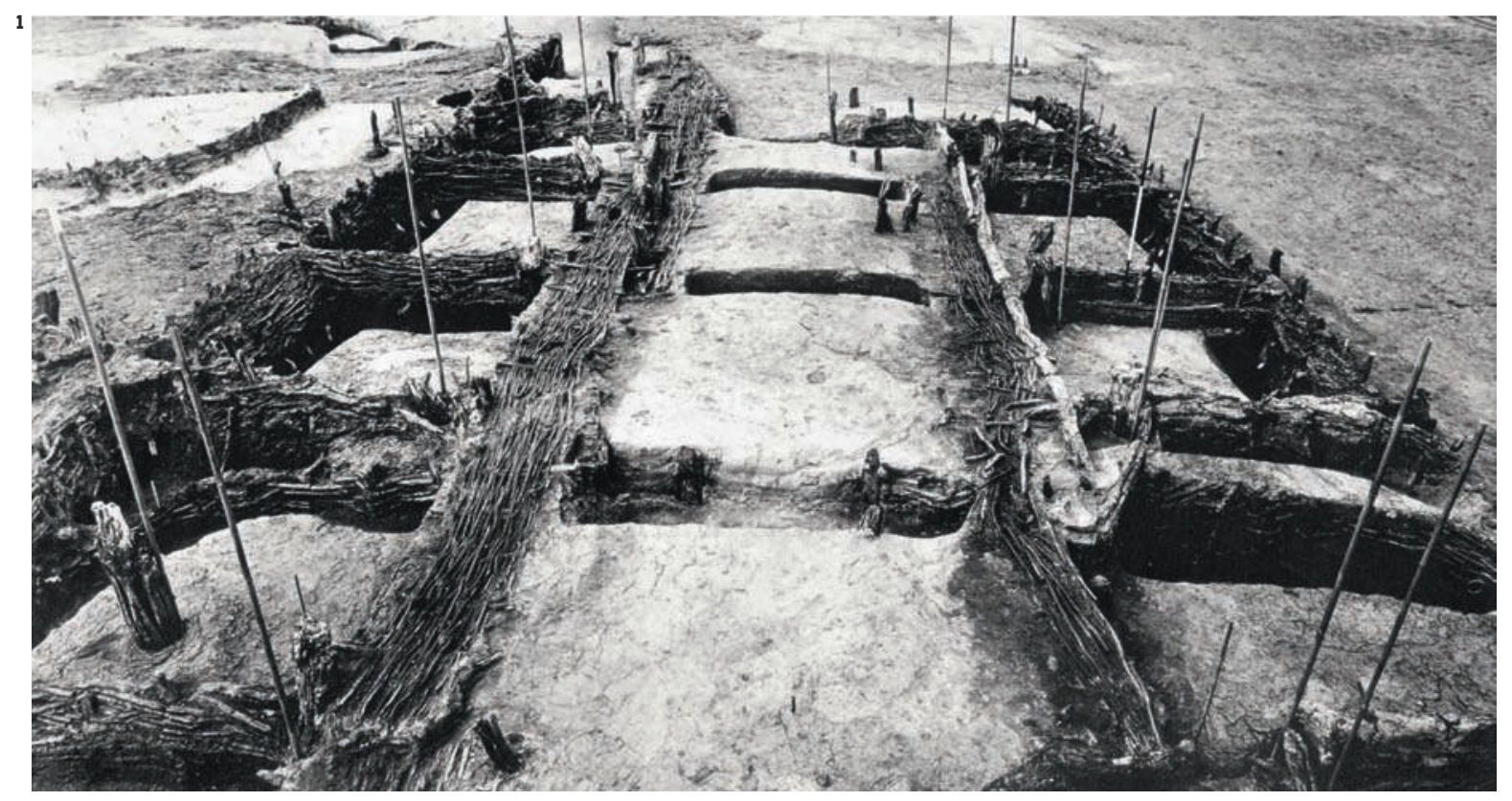

1. Une maison-étable en bois et torchis, préservée en élévation dans le « terp»"

d'Ezinge (Pays-Bas).

2. Montage de quelques plans

typiques de maisons à étables

excavées (dites « potsta/n) et

proposition de reconstitution

sur la base de l'exemple $n^{\circ} 1$

(Flandres, Belgique).
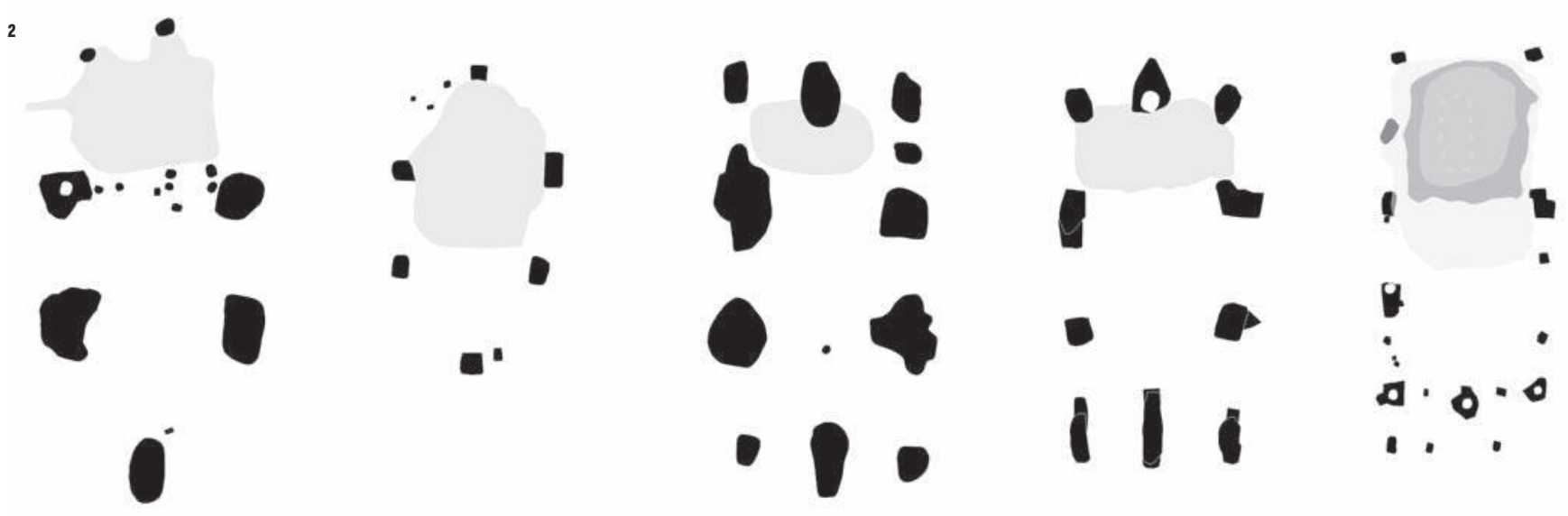

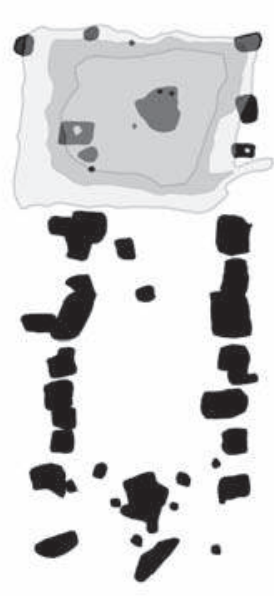

6

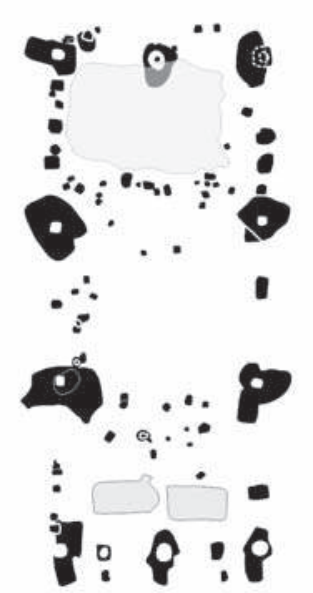

7
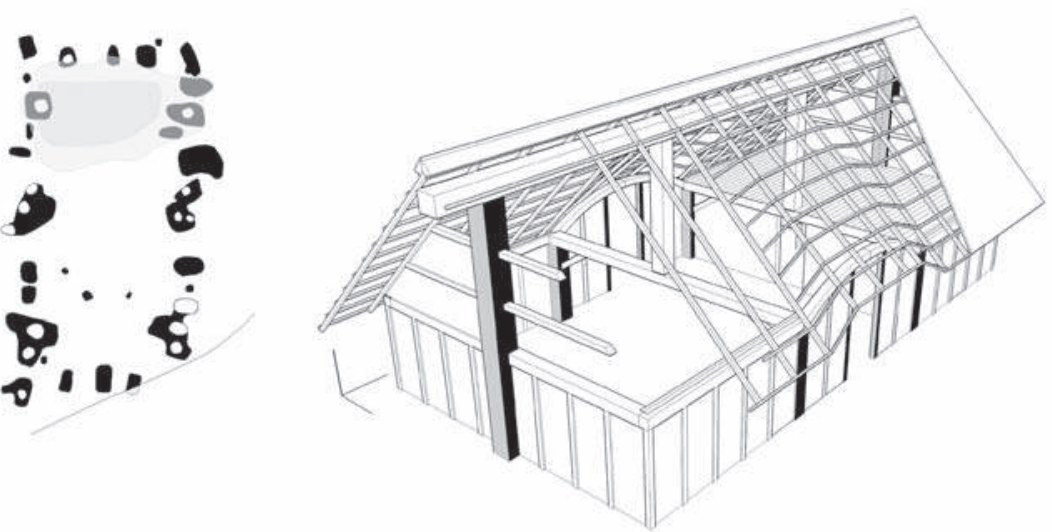

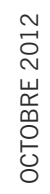

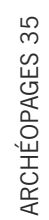



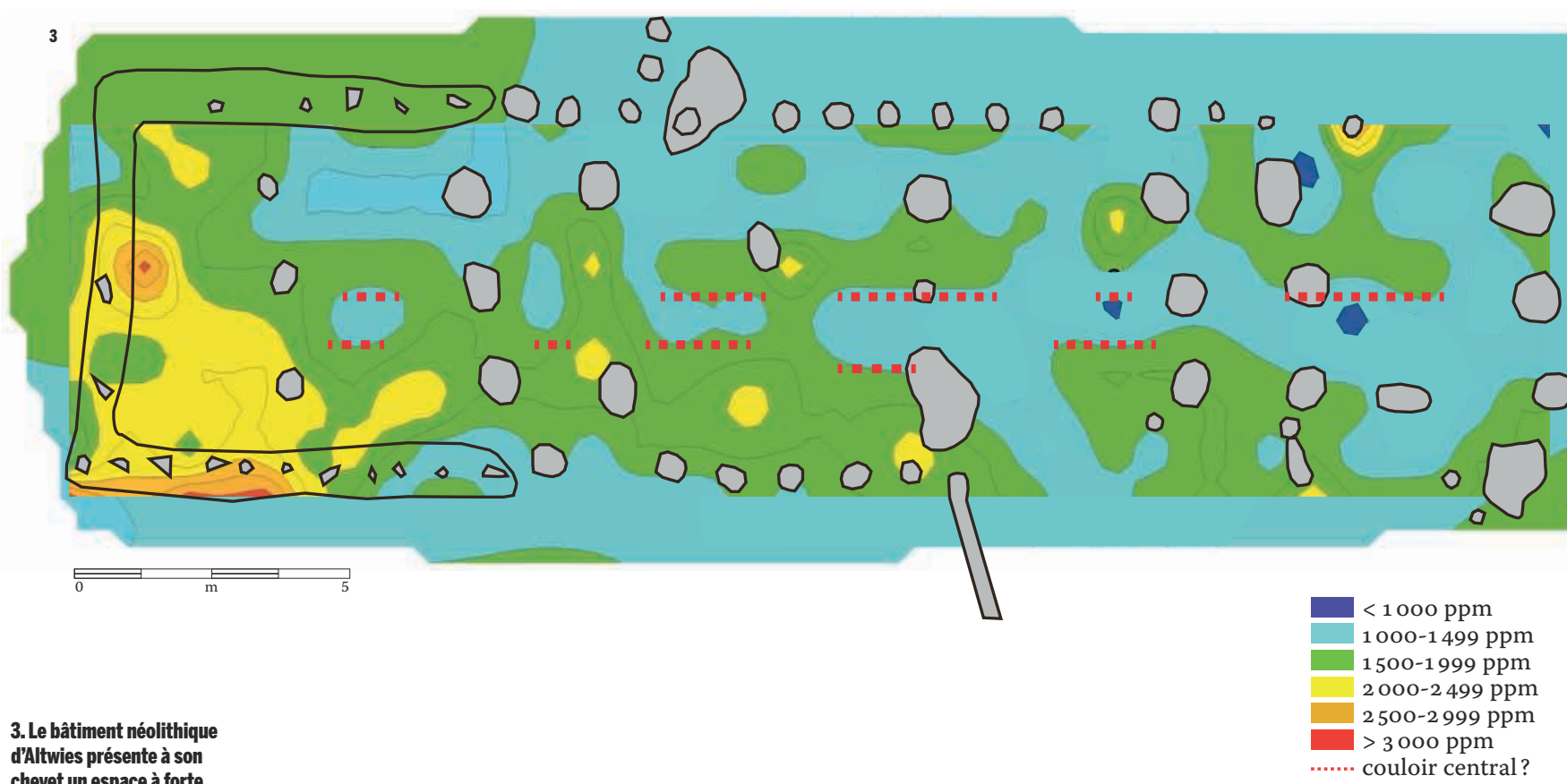

3. Le bâtiment néolithique d'Altwies présente à son chevet un espace à forte accumulation de phosphore lié à la matière organique, vraisemblablement issue d'excréments. Un couloir axial plus pauvre en phosphore montre une partition de l'espace interne proche des cas plus récents de bâtiments-étables (remerciements : Centre

National de Recherche Archéologique, Grand-Duché du Luxembourg).

4. Exemple néolithique sans forte accumulation de phosphore : I'enrichissement interne du bâtiment de Sauchy-Lestrée est localisé et limité (moins de 1400 ppm), sans lien avec la matière organique.

L'encaissant du bâtiment est un limon. II n'est pas érodé et la pénétration du phosphore est faible comme le montre un profil de référence à l'aplomb du bâtiment (remerciements à l'équipe Inrap Canal Seine-NordEurope).

Sauchy-Lestrée, bâtiment 80o: taux de phosphore total

\section{0}

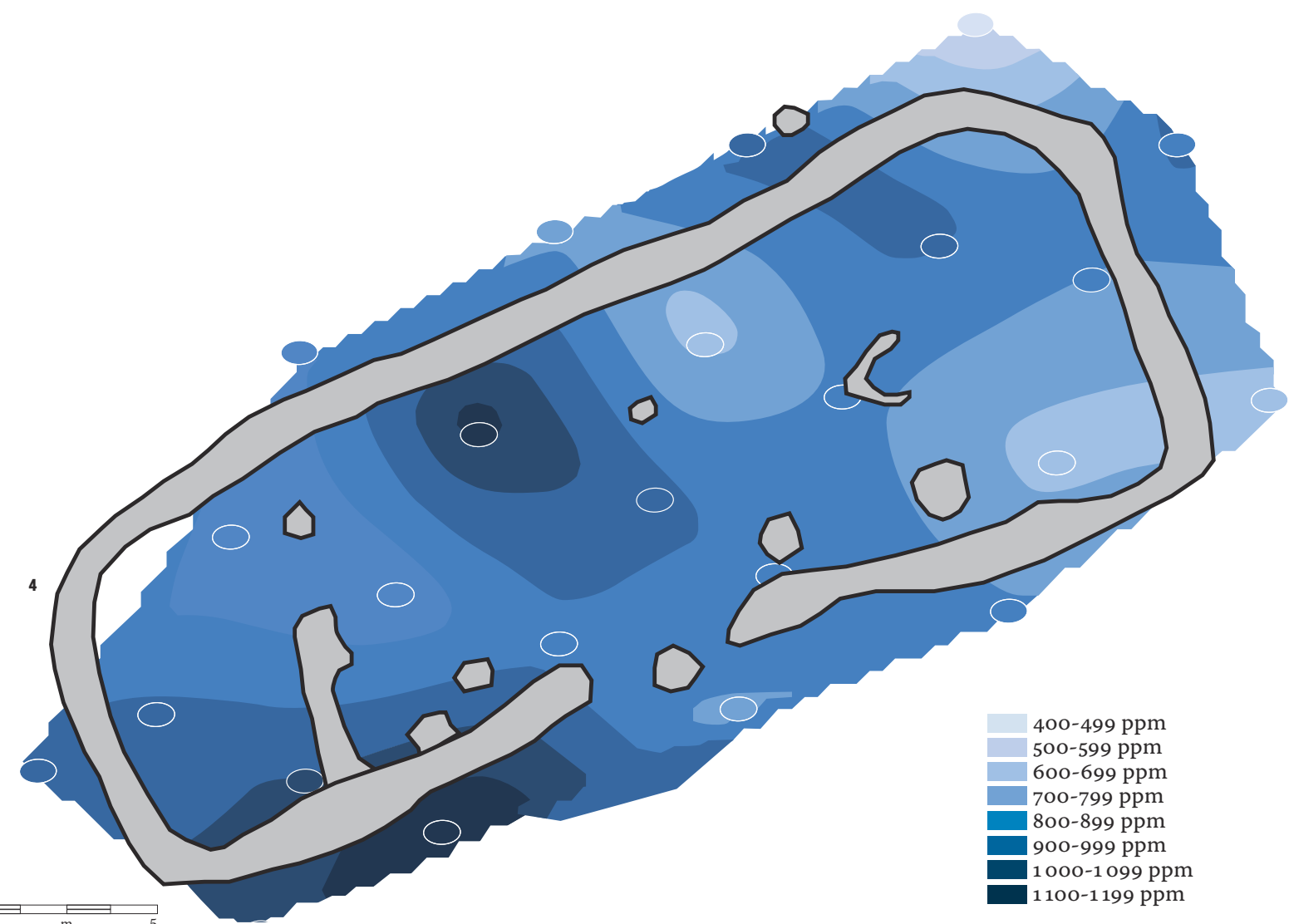


particulièrement bien conservées, dans lesquelles on observe une division interne en deux ou trois modules fonctionnels d'après l'emplacement de poteaux, souvent conservés sur des hauteurs considérables, ainsi que des «stalles» dans l'un des modules (Van Giffen, 1936).

Après la Deuxième Guerre mondiale et jusqu'aux années 1990, la recherche s'est intensifiée, surtout dans les régions côtières et sur les sols sablonneux plus méridionaux des Pays-Bas et du nord de la Belgique. Au cours des dix dernières années, ces maisons ont été découvertes en grand nombre en Belgique, surtout sur les sols sablonneux et même dans la tourbe de la plaine côtière de Flandre et des régions adjacentes du sud-ouest des Pays-Bas (De Clercq, 2011). Sans doute est-ce la région où ce phénomène est le mieux documenté avec quelques centaines de plans de maisons-étables en bois, d'époque surtout gallo-romaines.

Les maisons-étables ont subi une évolution remarquable, accélérée à partir de la conquête romaine de la Gaule. Dès le deuxième âge du Fer, on distingue le plan typique des maisons-étables à deux nefs de type «Haps», souvent à entrées séparées pour hommes et animaux. Très tôt à l'époque gallo-romaine, probablement à la suite des évolutions agro-économiques et fiscales, ces maisons se sont transformées en des constructions à deux nefs, souvent plus robustes, avec des poteaux porteurs lourds et profonds. Ce plan de type «Alphen-Ekeren» subit lui aussi des changements considérables : dans le nord-ouest de la Gaule, l'architecture se simplifie encore avec, souvent, une nef, un recours plus courant aux charpentes et donc l'installation de greniers. Des poteaux plus lourds ou en surplus dans la partie étable visent à la renforcer. L'étable se situe dans la partie septentrionale de la maison et tend à être plus large après le milieu du $\mathrm{II}^{\mathrm{e}}$ siècle. Bien qu'il soit encore difficile d'établir si ce phénomène d'étable excavée est le résultat d'un processus d'enlèvement des déjections hiver après hiver ou s'il correspond à un creusement initial intentionnel, il démontre que les fermiers ont développé un système maîtrisé d'accumulation des déjections animales pour fertiliser leurs champs. Il reste à confirmer que ces excavations témoignent dès cette époque d'une préparation de fumier par mélange d'un horizon végétal, prélevé dans la nature, avec des excréments et de la création, par ce moyen, de sols fertiles du type «plaggen.»

Pour l'époque mérovingienne, les plans de maisons de type «saxon», découvertes en Angleterre ainsi que le long de la côte flamande, montrent également une division interne en trois modules avec parfois des entrées séparées comme dans les siècles précédents (Hamerow, 1999 ; Hollevoet, 2007). De même, les plans de maisons allongées, bien documentées notamment par H. Zimmermann sur les sites des embouchures de la Weser et de l'Elbe, mais aussi dans le nord des Pays-Bas, au Danemark et en Norvège, montrent une continuité dans l'organisation fonctionnelle des maisons en bois, comme dans les maisons de type Odoorn et Gasselte A (entre le $\mathrm{V}^{\mathrm{e}}$ et $\mathrm{x}^{\mathrm{e}}$ siècle) (Waterbolk, 2009). Après l'époque carolingienne, la reconnaissance de modules fonctionnels de type étable devient beaucoup plus difficile à appréhender du point de vue structurel et typofonctionnel. En Flandres et aux Pays-Bas, apparaissent des maisons à trois nefs vers 900 - qui perdurent dans certaines régions jusqu'au $\mathrm{XIX}^{\mathrm{e}}$ siècle -, pour lesquelles on possède peu de preuves en faveur d'une partie dédiée à la stabulation. Néanmoins, quelques plans de maisons à trois vaisseaux du type Gasselte $\mathrm{B}$ montrent des zones de stabulation excavées dans la première et la troisième nef, soit entre les parois et les poteaux porteurs lourds, comme cela est encore attesté sur quelques peintures du XVIII ${ }^{\mathrm{e}}$ siècle. En outre, l'étude pédologique des couches arables des champs et les observations historiques montrent des systèmes d'accumulation de fumier et de stabulation dans les maisons du bas Moyen Âge et ultérieures (Spek, 1992, p. 72-91; Langohr, 2001, p. 103-118).

\section{L'apport des sciences naturelles}

Cet état des lieux montre la nécessité de recourir en France, comme cela a été fait dans des régions plus septentrionales, à un plus grand nombre de grilles de lecture pour préciser les activités dans les bâtiments fouillés. De nombreux exemples de stabulation animale supposée sont mentionnés dans la littérature, mais ils ne sont le plus souvent pas argumentés de manière détaillée, ni localisés avec précision dans les ensembles de bâtiments à vocation agricole comme pour la plupart des bâtiments annexes des cas antiques.

Utilisées avec prudence, certaines approches environnementales peuvent apporter une argumentation indépendante de l'approche archéologique. Parmi celles utilisées avec succès dans le nord de la France, on peut citer la micromorphologie, les tests et analyses chimiques, la mesure de paramètres magnétiques (Hulin et al., à paraître), la résistivité électrique, la carpologie, les études des phytolithes, l'archéozoologie ou encore la parasitologie. Le croisement des informations obtenues avec celles récoltées par l'archéologue permet d'approcher l'activité humaine sur un site. Dans ce but, grâce à un projet PAS de l'Inrap, les environnementalistes se proposent de collecter les informations environnementales afin d'en faire une synthèse sous forme de cartes de répartition associées à un SIG.

\section{La cartographie du phosphore et la pédologie}

La cartographie du phosphore a été privilégiée à l'Inrap depuis quelques années, au début sous l'impulsion de Valérie Gonzalez et d'Hervé Guy, et surtout à la suite de l'expérience effectuée sur le site de la chapelle Saint-Nicolas à Saint-Bricesous-Forêt (Val-d'Oise) par Vanessa Rouppert. C'est la méthode le plus facilement applicable 

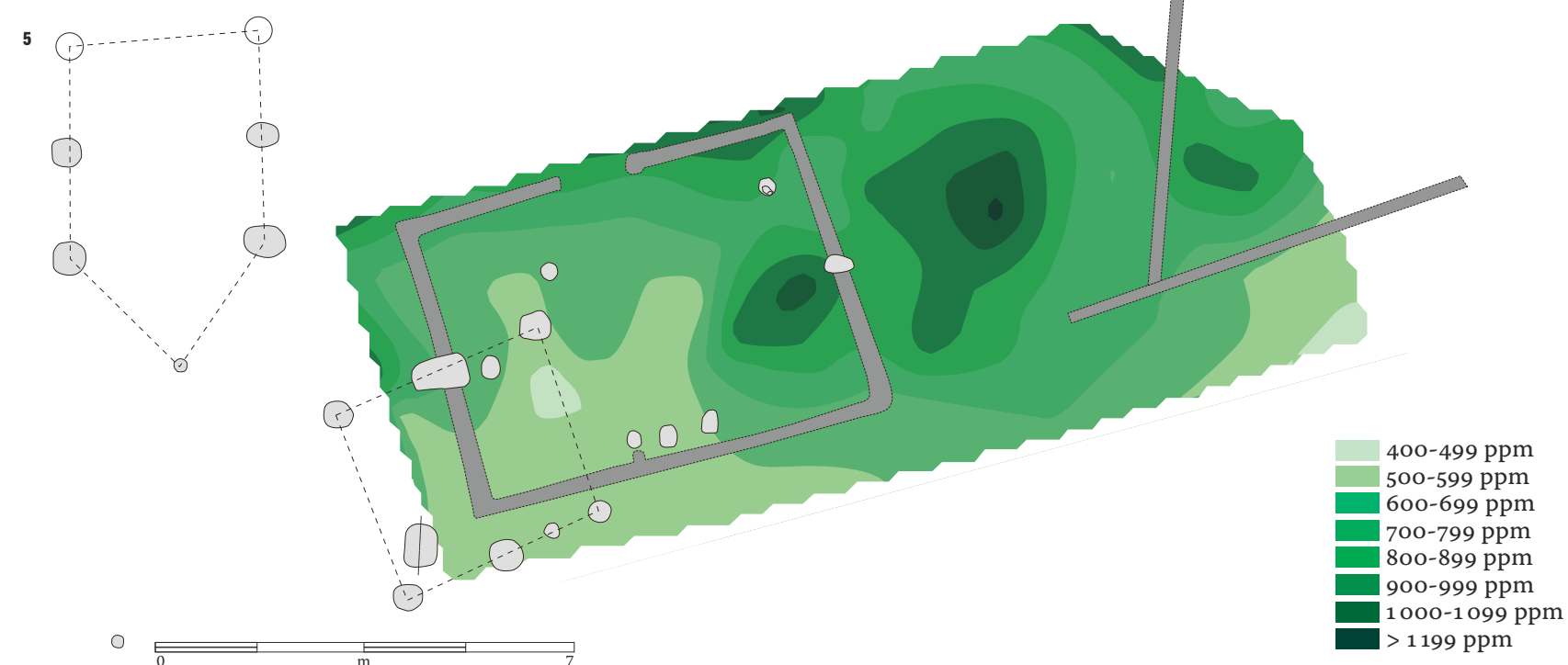

5. Exemple protohistorique phosphore:le bâtiment laténien $A / E$ de

Champfleury présente des taux de phosphore un peu plusélevés (650-1600 ppm contre 600-1400 ppm dans les environs) compatibles avec de l'élevage. La cour délimitée par le bâtimentet deux fossés est la plus riche en phosphore (1000-2000 ppm).Ils'agit d'une sorte de cour délimitée par le

bâtiment et par deux fossés. 6. Exemple protohistorique sans forte accumulation de phosphore:l'intérieurdu bâtiment laténien à pans coupés de Pont-sur-Seine/ Marnay-sur-Seine montre une absence d'accumulationde phosphore, alors que l'on détecte un léger enrichissement externe de tous côtés.

-999 ppm 1 0oo-1124 ppm 1125-1249 ppm 1250-1374 ppm 1375-1 $499 \mathrm{ppm}$ 1500-1624 ppm 1625-1774 ppm $>1775$ ppm - effet de paroi 
à un grand nombre de sites et directement associée au plan d'une unité architecturale ou d'une autre occupation. Le phosphore permet d'approcher au mieux les activités liées à l'élevage, au stockage ou à la présence d'ossements. Cet élément chimique est présent dans les tissus de tout être vivant (ADN, parois cellulaires, métabolites) et particulièrement concentré dans les excréments et les biominéraux composant les ossements et les coquilles. Pour réaliser une cartographie du phosphore, de nombreux échantillons de sédiments sont prélevés lors de décapages archéologiques et dans certaines structures en creux. Leur abondance en phosphore est testée à l'aide d'un réactif avant d'être visualisée sur un plan-masse. Des échantillons pertinents sont sélectionnés pour les analyses au laboratoire, afin de chiffrer l'accumulation, de préciser sa nature et de permettre la comparaison objective entre structures et sites.

Menées de longue date en Europe, et régulièrement depuis les débuts de l'archéologie préventive en France, les cartographies du phosphore ont été systématisées le long du tracé du canal Seine-NordEurope et en Nord-Picardie depuis 2009. Cela a été possible en associant les compétences de trois chercheurs $^{2}$ et avec le soutien des archéologues et de leurs responsables respectifs ${ }^{\mathbf{3}}$. Le caractère systématique consiste dans la démarche pédologique, la sélection raisonnée de tous les sites à haut potentiel, l'application de programmes de présentation et d'interpolation dès la phase de tests, le traitement statistique des données, l'unification des codes colorimétriques utilisés pour les plans des cartographies et enfin le croisement avec la cartographie de paramètres magnétiques. Ceux-ci nous renseignent sur la teneur et la composition des oxydes de fer dans le sol, elles-mêmes étroitement liées aux activités humaines. Chauffe et matière organique constituent les deux facteurs les plus importants dans l'accumulation particulière des oxydes de fer en contexte archéologique. Associées à des outils informatiques adaptés, ces démarches ont pu être aisément rapportées de l'échelle de la structure à celle du site (enclos, village...).

En pratique, même si la pédologie semble facile à mettre en œuvre, il faut tenir compte de nombreux facteurs. Le plus important est le contexte pédologique général du site (affleurements géologiques, végétation, nappes phréatique et perchée, impacts anthropiques antérieurs et postérieurs à l'occupation...). Pour chaque échantillon, il s'avère primordial de tenir compte des facteurs physiques, chimiques et biologiques qui affectent le comportement du phosphore, rendant indispensable une connaissance approfondie de la pédologie.

Cette prise en compte intervient à trois niveaux. D'abord sur le terrain, afin d'estimer de la faisabilité de l'étude et sélectionner les secteurs. Ensuite à l'interprétation, afin de comprendre les taux observés selon le type d'encaissant ou le taux de bioturbation constaté sur le site. Enfin, lors de la synthèse des résultats, afin de comprendre le lien entre sol, pratique humaine et conservation du phosphore sur le site et jusqu'au niveau d'un terroir. Une fois ces précautions prises, il est possible d'interpréter nos résultats et d'émettre des hypothèses à confronter aux données archéologiques. Dans certains cas, cela a permis de favoriser la possibilité d'un espace à forte accumulation d'excréments [ill. 3], dans d'autres celle d'une forte accumulation d'ossements [ill. 4] et dans d'autres encore d'exclure toute activité polluante de ce type [ill. 5], sans risquer une confusion provoquée par l'accumulation ou au contraire la perte de phosphore d'une autre époque que celle de l'occupation.

\section{La recherche de la place des animaux dans les maisons}

Les exemples suivants illustrent ce croisement de données. Deux sont pris par période, le premier montrant un espace à forte accumulation de phosphore, le second excluant toute possibilité d'accumulation ancienne. Il ne s'agit pas ici d'un inventaire des études menées.

Pour le Néolithique, nous traiterons du bâtiment 7 du site d'Altwies (Néolithique ancien) [ill. 3], situé dans le Grand-Duché de Luxembourg, près de la frontière française, et du bâtiment 800 de Sauchy-Lestrée (Néolithique final), dans le Pasde-Calais. Plusieurs indices, dans le bâtiment 7 , se rapprochent de ce que l'on observe dans les bâtiments-étables postérieurs. D'abord, l'accumulation de phosphore limitée à une aire du bâtiment, en particulier dans le chevet renforcé, est plus importante que ce qui est connu pour les autres bâtiments du Néolithique ou de l'âge du Bronze étudiés dans la région (Fechner et al., 2011). Ensuite, cette accumulation de phosphore est associée à de la matière organique comme le révèle l'étude de la susceptibilité magnétique du sol ${ }^{5}$. L'encaissant sur lequel est implanté le site est un limon décarbonaté sur une faible profondeur. La marne est située à moins d'un mètre sous le niveau du sol, ce qui aurait pu avoir une influence sur les teneurs en phosphore observées dans le bâtiment, assez élevées en effet pour un site néolithique (entre 1100 et 2100 ppm de P total). Or, les taux dépassant les 1500 , voire $2000 \mathrm{ppm}$, sont également observés dans cinq autres maisons du Néolithique ancien, dont quatre implantées sur des limons profondément décarbonatés. Sur ce type d'encaissant, la rétention de l'eau par le sol est plus faible qu'à Altwies et n'a pu avoir une influence sur les teneurs en phosphore.

Soulignons que les accumulations de phosphore correspondent, pour les quatre bâtiments traités, à des pics de susceptibilité magnétique, donc vraisemblablement à de la matière organique. Ce trait récurrent pourrait plaider pour une origine organique des accumulations de phosphore (par opposition aux ossements). La stabulation
Fechner et Viviane Clavel.

Prilaux, Laurent Sauva

Véronique Kasimirczak.

4. Responsables d'opération
Catherine Jost, Anne

Hauzeur, Foni Le Brun-

environnementales :

Robert Baes, Kai Fechner.

5. Réalisée par Jozef Hus,

Institut royal de

météorologie de Belgique 


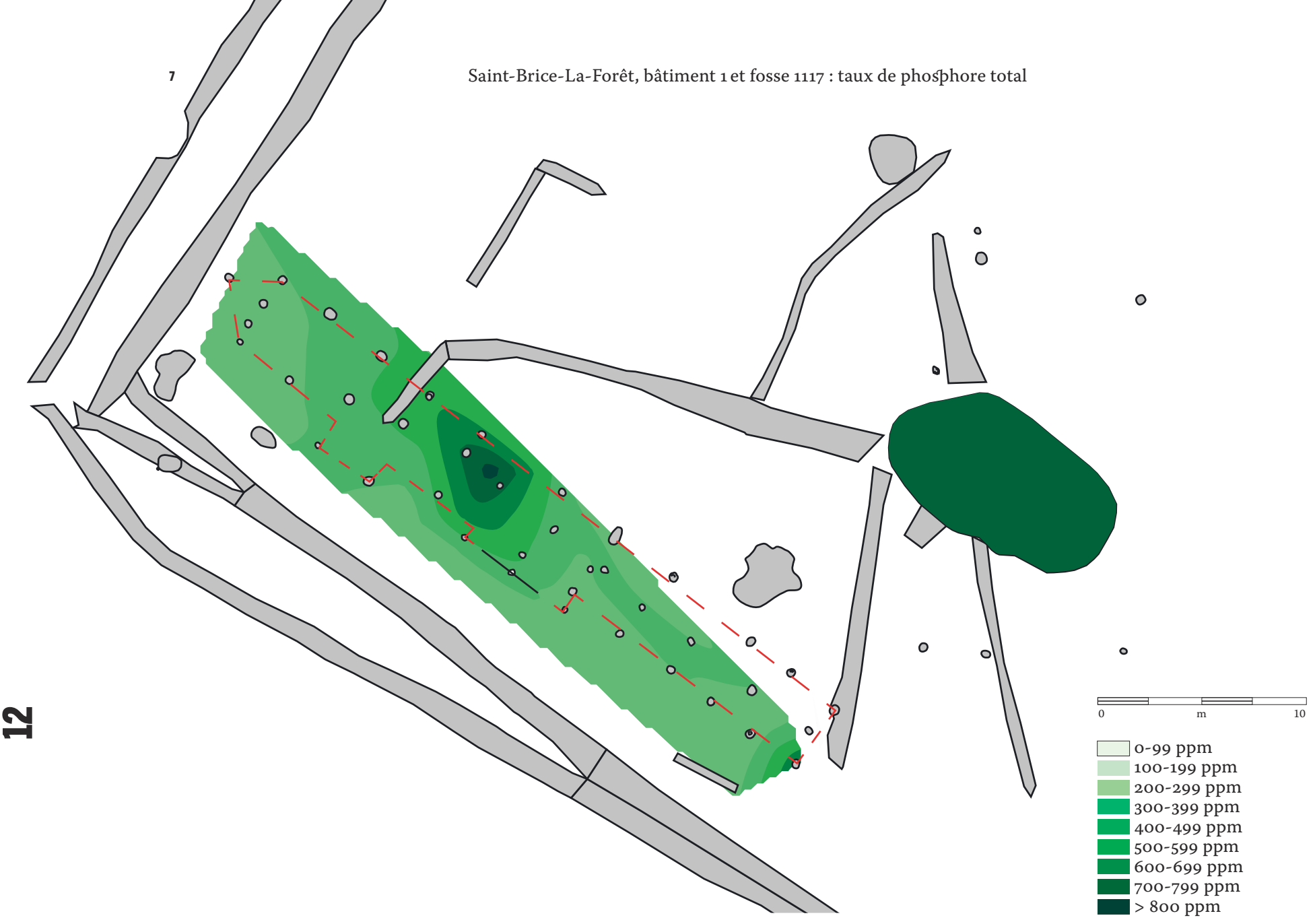

7. Exemple gallo-romain de Saint-Brice-sous-Forêt avec forte accumulation de phosphore : les valeurs du bâtiment (250-900 ppm) dépassent celles de l'enclos (250-500 ppm). Elles sont influencées par des limons à bioturbation profonde et intense, à profondeur de décarbonatation inférieure à $1 \mathrm{~m}$. Hors contexte de stabulation, les valeurs passent sous les $650 \mathrm{ppm}$.

8. Exemple gallo-romain sans forte accumulation de phosphore: dans le bâtiment annexe de la villa de Merbes-le-Château, la répartition des résultats montre des concentrations de phosphore faibles, sauf points isolés au sommet de l'encaissant.

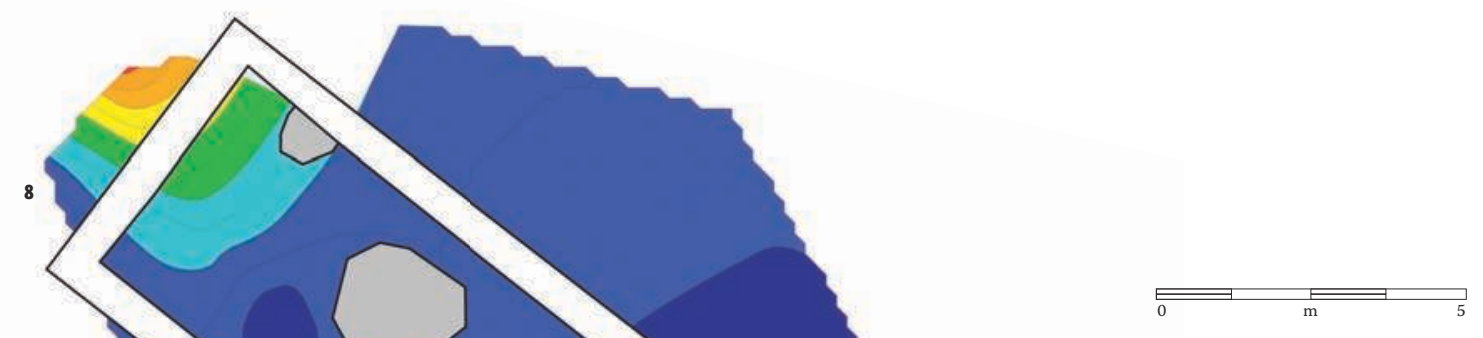

près de nul

extrênement bas très bas bas

moyen

moyen élevé

élevé

très élevé

extrênement élevé 
ne reste qu'une hypothèse et le lien entre zones plus riches en phosphore et parois renforcées n'est pas un élément retenu par les archéologues. En effet, d'autres sources d'accumulation de phosphore restent envisageables pour ces bâtiments, comme le torchis ou le stockage de végétaux riches en phosphore.

L'étude menée sur le bâtiment 8 oo de SauchyLestrée ${ }^{\mathbf{6}}$ [ill. 4] n'a permis d'observer des effets de paroi bien marqués que lors de la cartographie des tests de phosphore?. Tout comme les quatre autres bâtiments du Néolithique final, traités selon les mêmes méthodes et localisés dans le même département et sur les mêmes sols, celui-ci présente des indices incompatibles avec une interprétation comme lieu de stabulation. L'hypothèse la plus plausible est celle d'une occupation de l'encaissant causée par les activités domestiques (cendres, torchis, restes de repas, ....). L'étude de la susceptibilité magnétique montre des valeurs plus élevées à mi-longueur du bâtiment. Cela demande une explication, mais pourrait être lié à la présence plus abondante de restes carbonisés. Cette observation est importante car certains bâtiments allongés du Néolithique final présentent, à ce niveau, un agencement des trous de poteaux interprété comme un grenier intérieur ou, du moins, comme un espace intérieur surélevé. La première hypothèse pourrait être soutenue sur le site de Méaulte (Somme) par l'agencement particulier des trous de poteau et la répartition des macrorestes à l'intérieur (Joseph et al., 2011). Un autre de ces cas est marqué, sur le même emplacement, par un vide dans la cartographie du phosphore, bien délimité par les trous de poteaux (Aire-sur-la-Lys ${ }^{\mathbf{8}}$ ).

Le bâtiment $\mathrm{n}^{\circ} \mathrm{A} / \mathrm{E}$ du secteur 1 de Champfleury « La Fosse aux fromages » (Marne) est daté de la transition entre La Tène finale et l'époque gallo-romaine [ill. 5] ${ }^{\mathbf{9}}$. Les taux observés et la pauvreté en ossements font supposer une source organique à l'accumulation de phosphore. L'étude de la susceptibilité magnétique limitée au secteur 4 montre une corrélation entre taux de phosphore élevés et matière organique. Cet ensemble est en opposition avec les longs bâtiments sur sablières du secteur 1 , appauvris en phosphore par rapport à leurs alentours. Les valeurs rappellent celles de l'intérieur de l'enclos synchrone du secteur 4, sans doute associé à de l'élevage (Fechner, 2011).

À Pont-sur-Seine/Marnay-sur-Seine (Aube), le secteur protohistorique a livré un bâtiment au plan hexagonal dit «à pans coupés » [ill. 6] ${ }^{\mathbf{1 0}}$ qui témoigne d'une tendance observée sur une demidouzaine d'autres bâtiments similaires traités avec les mêmes méthodes dans le nord de la France ${ }^{\mathbf{1 1}}$. La cartographie du phosphore livre l'image d'un bâtiment sans aucune activité polluante et permet d'apporter des informations architecturales. En effet, la zone sans phosphore étant strictement limitée à l'aire circonscrite par les poteaux, ces derniers ne peuvent faire partie que de la structure externe du bâtiment et ne sont pas des poteaux porteurs internes au sein d'une structure plus large.

Les enclos gallo-romains de la Chapelle SaintNicolas à Saint-Brice-sous-Forêt (Val d'Oise) ont livré un bâtiment sur poteaux plantés [ill. 7] daté du Haut-Empire ${ }^{\mathbf{1 2}}$. Sa caractérisation comme étable à bovins s'appuie sur des critères morphométriques, établis par rapport à un référentiel relevant de la logique agronomique, ainsi que sur des marqueurs chimiques (richesse et répartition en phosphore) et des indices mobiliers (auge, nombreuses pierres à aiguiser, etc.) à défaut d'indices zoologiques, parasitologiques ou botaniques qui ont également été recherchés. De plus, l'étude chronostratigraphique a permis d'établir une liaison fonctionnelle entre la partie la plus riche en phosphore du bâtiment et le fond de la grande double fosse adjacente, par l'entremise de fossés de connexion. Cette fosse possède des caractéristiques stratigraphiques, chimiques et typofonctionnelles qui favorisent l'hypothèse d'une fosse à purin et à fumier, cohérente dans cette partie orientale de l'enclos (Rouppert et al, 2011, p. 133-142).

Pour l'époque gallo-romaine toujours, le site de Saint-Étienne-au-Mont (Pas-de-Calais) présente un bâtiment dont une des deux partitions semble avoir accueilli des bovins (Bazile, Clotuche, à paraître). Ces deux cas illustrent parfaitement l'intérêt d'une démarche pluridisciplinaire et ont ouvert la porte à la recherche d'autres bâtiments de stabulation du grand bétail dans le nord de la France.

Le site de Merbes-le-Château est situé en province du Hainaut, en Belgique, à quelques kilomètres du Nord ${ }^{\mathbf{1 3}}$. Un bâtiment annexe très allongé, et légèrement surcreusé a été mis au jour, situé dans la pars urbana, devant et à la perpendiculaire du bâtiment principal de la villa gallo-romaine [ill. 8]. L'hypothèse d'une étable a pu être exclue par l'absence d'accumulation de phosphore, permettant de rechercher d'autres solutions (jardin aménagé?). Cet exemple illustre l'apport des sciences de la terre comme qu'étape indispensable à l'interprétation.

En bordure du double enclos gallo-romain de Lesquin (Nord) ${ }^{\mathbf{1 4}}$, un autre bâtiment allongé, sur sablières, ne présente aucun lien avec du phosphore, contrairement aux enrichissements voisins, nettement associés aux enclos.

Deux des cinq cas étudiés ( $n^{\circ} 6147$ et 6225 ) parmi les fonds de cabanes et bâtiments creusés de Pontsur-Seine/Marnay-sur-Seine ${ }^{\mathbf{1 5}}$ livrent plusieurs indices en faveur de l'hypothèse de fonds de cabane ayant accueilli un animal [ill. 9]. À Roissy-en-France (Val d'Oise) ${ }^{\mathbf{1 6}}$, l'hypothèse de bergeries a été proposée pour trois bâtiments médiévaux sur la base de la typofonctionnalité et de l'étude des textes agronomiques. Pour les deux exemples du XIII ${ }^{\mathrm{e}}$ siècle et des $\mathrm{XV}^{\mathrm{e}}-\mathrm{XVII}^{\mathrm{e}}$ siècles, la démarche a combiné les observations d'accumulations de phosphore, l'analyse micromorphologique, l'étude des ossements et celle des phytolithes (Dufour, 2007 ; Verdin, à paraître) ${ }^{\mathbf{1 7}}$. Il en résulte que les céréales et leurs sous-produits, associés à d'autres 
Profil P2o

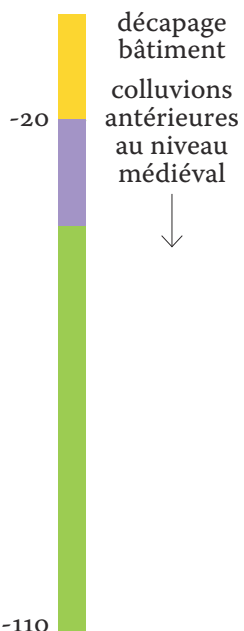

Profil de référence

abours

$-27 \mathrm{~cm}$

colluvions

sol encaissant en place

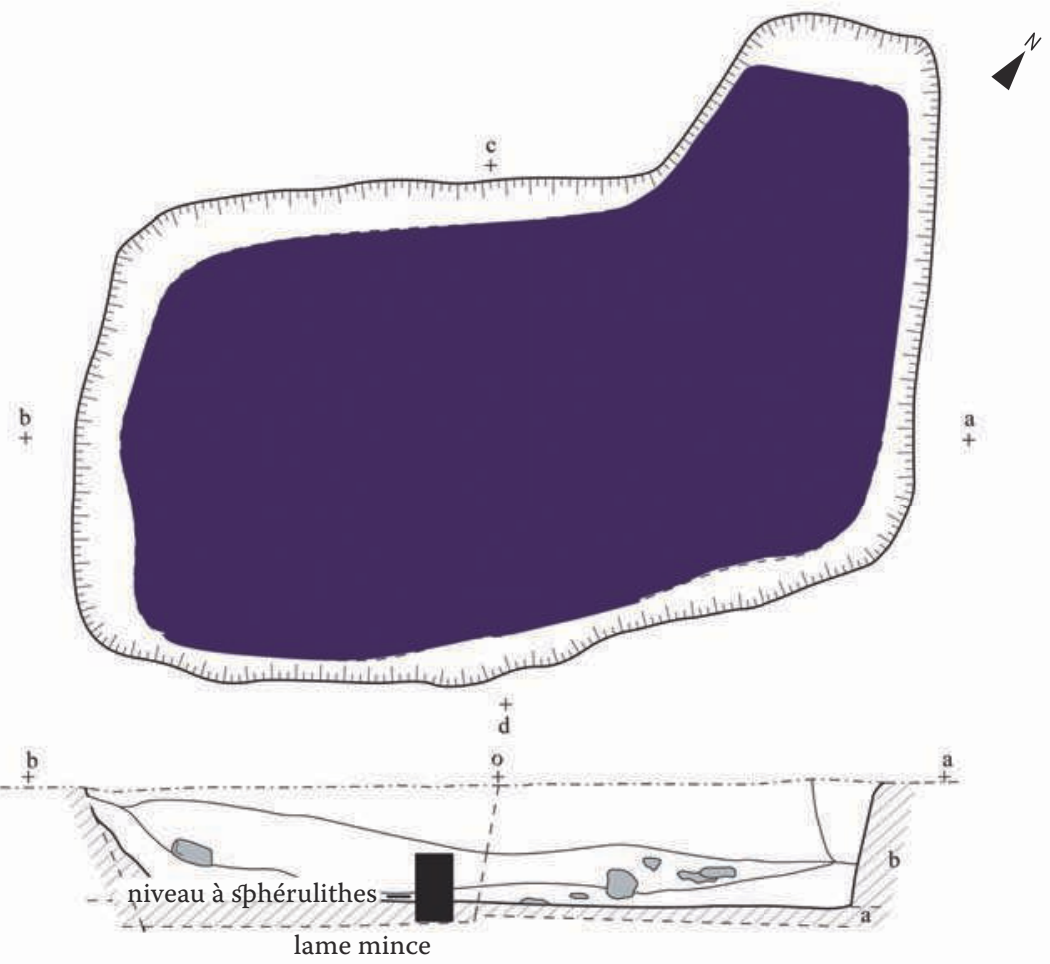

Plan du fond
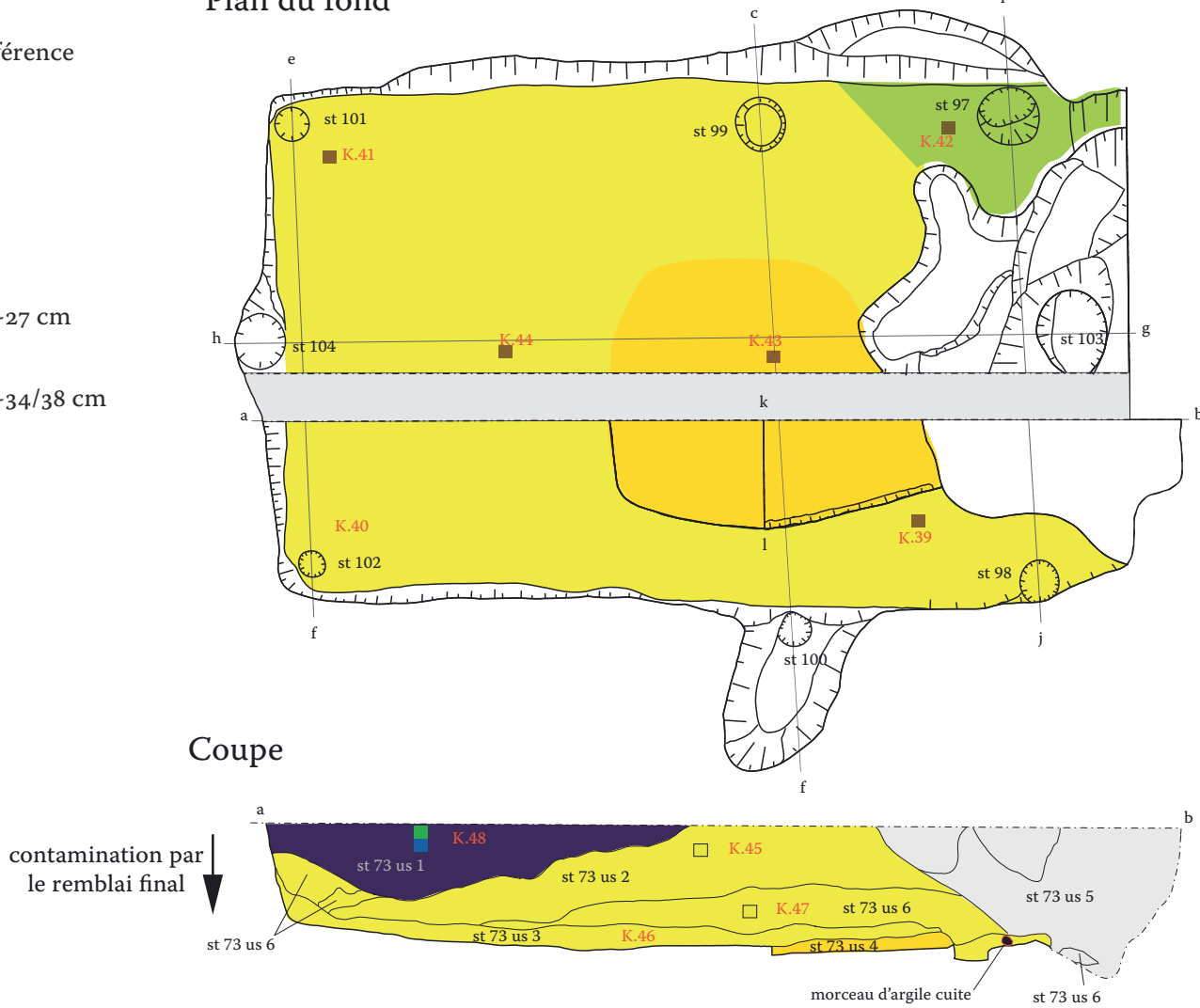

0-299 ppm $300-599 \mathrm{ppm}$ 6oo-899 ppm 900-1199 ppm 1200-1 499 ppm 1500-1799 ppm 1800-2 099 ppm 2100-2 $399 \mathrm{ppm}$ 2 400-2 699 ppm 2700-2 999 ppm $3000-3299 \mathrm{ppm}$ 3300-3 599 ppm $3600-3899$ ppm $3900-4,199 \mathrm{ppm}$ 4. 200-4 499 ppm 4500-4799 ppm $4800-(+)$ ppm

9. Exemple d'un fond de cabane du premier Moyen Âge avec forte accumulation de phosphore : à Pont-surSeine/ Marnay-sur-Seine, la fine couche de fond est en outre riche en phytolithes de graminées et en sphérolites (formation calcaires caractéristiques de la digestion des herbivores, voire des ovi-caprins) en lame mince (indiquée par un rectangle) (remerciements à l'équipe Inrap Grand Est Nord).

10. L'absence

d'accumulation de

phosphore du fonds de cabane de Méricourt montre que ces structures ne servaient pas à une activité pastorale. Des taux exceptionnels sont uniquement présents dans le comblement final du fait d'ossements altérés. Le fond, par contre, montre des taux moyens et peu constants, dus à l'influence des comblements supérieurs (remerciements à l'équipe Inrap Nord-Picardie). 
19. Fouille : Samuel Desoutter. Analyses :

Kai Fechner et Viviane Clavel. 19. Fouille :

Ludovic Notte.

20. Analyses : Kai

Fechner et Viviane Clavel. indices phytolithiques, permettent de déterminer le stockage des céréales et la stabulation pour les moutons et les porcs, dans les sols de basse-cour et dans les zones d'habitat. La comparaison des analyses chimiques avec les constituants organiques et phosphatés observés en lame mince permet d'affiner les critères déterminants des activités productrices de matière organique.

À Craywick (Nord) ${ }^{\mathbf{1 8}}$, c'est un espace extérieur aménagé entre deux fossés parallèles (cour?) et perpendiculaire à un bâtiment allongé qui montre des valeurs compatibles avec un espace de stabulation.

À Méricourt (Pas-de-Calais) ${ }^{\mathbf{1 9}}$, six fonds de cabanes ont fait l'objet d'une cartographie du phosphore et présentent une pauvreté du fond en phosphore malgré d'occasionnelles taches de phosphore visibles à l'œil nu [ill. 10] ${ }^{\mathbf{2 0}}$. Des bâtiments médiévaux allongés comme celui de Craywick frappent également par l'absence de phosphore, par opposition aux échantillons de référence prélevés au décapage sur ses quatre côtés.

Un projet PAS de l'Inrap, initié en 2011, a permis de produire une première carte de répartition des bâtiments ayant fait l'objet d'analyses pluridisciplinaires dans la frange nord de la France [ill. 11]. Ce projet est intitulé «Cartographie et base de données des structures et espaces interprétés de manière interdisciplinaire en Nord-Picardie. Un SIG pour les milieux ruraux de l'âge du Bronze au haut Moyen Âge en Nord-Picardie et dans les départements limitrophes ». Il traite d'autres structures archéologiques (horizons de surface anciens, fosses, fossés, structures de combustion, levées) qui font appel aux sciences environnementales pour préciser leur fonctionnement. Ces cartes de distribution peuvent être déclinées par contexte, par interprétation et par époque. En cela, elles sont le reflet d'un état d'avancement et d'une base de données en évolution. Il s'agit d'un outil de première synthèse des résultats environnementaux qui facilite les synthèses globales.

La réunion des deux approches menées par les archéologues et les environnementalistes permet un dialogue passionnant comme l'ont déjà montré les travaux de $\mathrm{H}$. Zimmermann dans les régions plus septentrionales : l'un ne peut souvent être aussi pertinent qu'il le souhaiterait sans l'apport de l'autre. À l'inverse, la collaboration permet une compréhension précise de l'utilisation des unités architecturales, comme pour les étables connues aux Pays-Bas, dans le nord de l'Allemagne et dans le sud de la Scandinavie à partir de l'âge du Bronze et pour les maisons-étables dès l'âge du Fer en Flandre (Belgique). Quelques cas de stabulation pour le nord de la France ont été présentés, en particulier pour les époques romaines (SaintBrice-sous-Forêt et Saint-Etienne-au-Mont) et médiévales (Pont-sur-Seine, Jossigny en Seine-etMarne, Épiais-lès-Louvres (Val-d'Oise) [cf. encadré p. 17] et Roissy-en-France). D'autres exemples présentés s'attachent à montrer l'efficacité de la cartographie du phosphore lorsqu'il s'agit d'exclure toute forme de stabulation pour certains bâtiments. Enfin, on met en évidence des résultats intéressants pour certains espaces externes (enclos, cours) potentiellement liés aux animaux (Champfleury à la Tène finale, Craywick au premier Moyen Âge). La question de la stabulation au Néolithique ancien est aussi posée. Aucun des cas ne serait parlant sans la prise en compte des facteurs pédologiques et post-dépositionnels.

L'avenir de la recherche sur le sujet passera inévitablement par la multiplication des études globales ainsi qu'une démarche de synthèse et de comparaison des données, notamment par les projets PAS de l'Inrap. Il sera particulièrement utile de systématiser les protocoles d'étude en vue de cette synthèse, comme sur le canal Seine-NordEurope et comme cela tend à se généraliser à l'Inrap. Dans les rares cas où la conservation des horizons de surface le permet, il est utile de passer à des observations, encore trop isolées, sur les parasites, les phytolithes, les sphérolithes. Enfin, en pratique, on ne saurait croiser efficacement ces résultats divers sans une discussion entre tous les partenaires (d'un chantier, d'une région...), échange que nous avons initié ici. In fine, c'est la prise en compte du contexte global et de toutes les autres composantes de la société agricole qui aboutira à une compréhension satisfaisante de ce pan de l'activité économique du passé. 


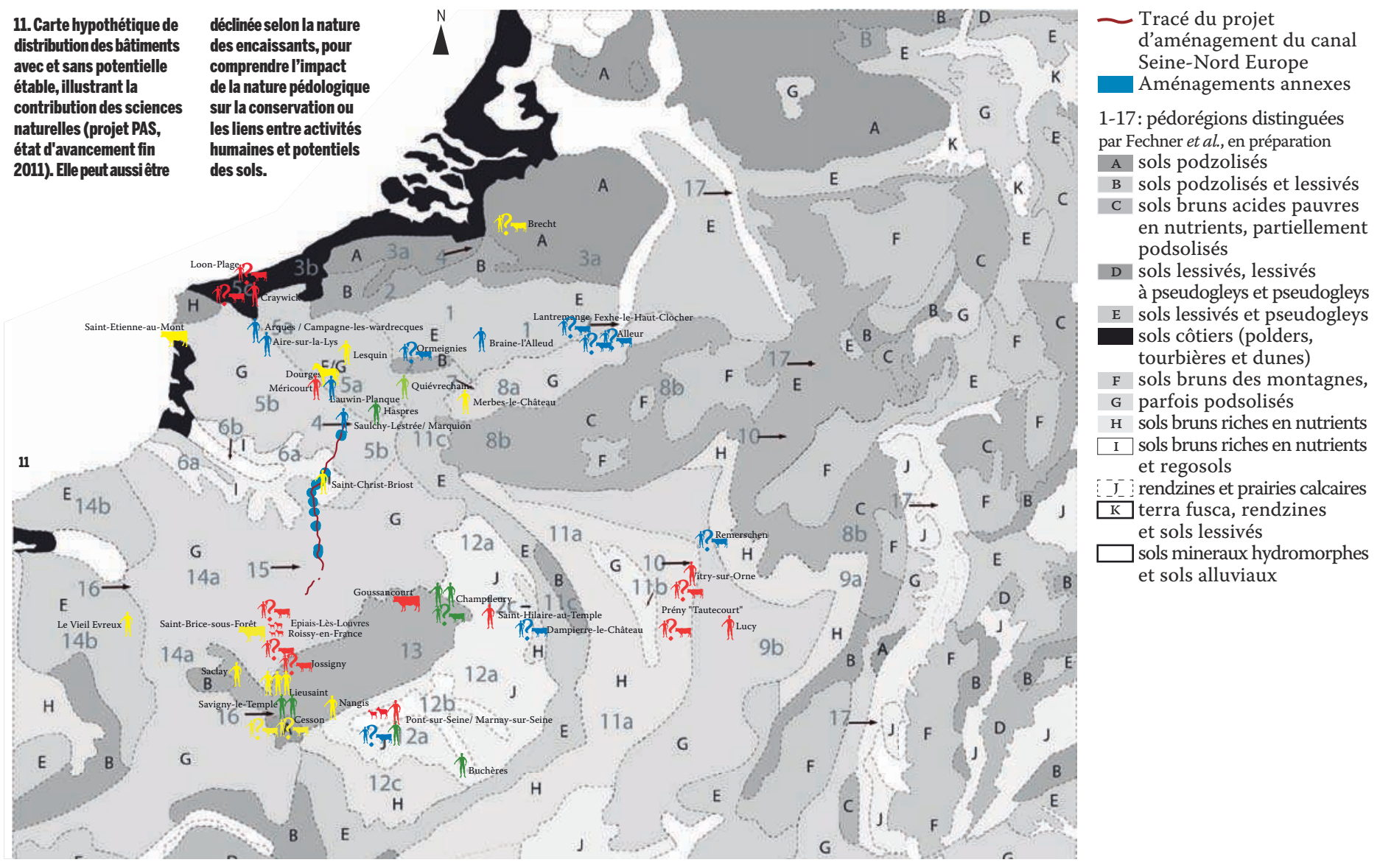

Interprétation des unités architecturales par périodes Néolithique âge du Bronze âge du Fer gallo-romain Moyen Âge

\begin{abstract}
† bâtiment peu ou pas N enrichi en phosphore

P. bâtiment avec partie fortement enrichie en phosphore (stabulation?)

An écurie étable

Th bergerie
\end{abstract}

\section{Références bibliographiques}

BAZILE F., ClOTUCHE R., à paraître, « $\mathrm{A} 3{ }^{\text {rd }}$ Century AD house construction at Saint-Étienne-au-Mont (Boulogne-sur-Mer, Pas-de-Calais)

Complementarities of chemistry and archaeology for a functional characterisation », in FECHNER K., DeVos Y., LEOPOLD M., VÖLKEL J. (ÉDS.),

Archaeology, soil-and life sciences applied to houses and gardens, Actes de l'European Association of Archaeologists's $12^{\text {th }}$ Annual Meeting, Krakow, 2006, vol. 2,. BAR, International Series.

Bostyn F., Martial E., Praud I. (DIR.), 2011, Le Néolithique du nord de la France dans son contexte européen : habitat et économie aux $4^{e}$ et $3^{e}$ millénaires avant notre ère, Actes du $29^{e}$ colloque interrégional sur le Néolithique, Villeneuve d'Ascq, 2-3 octobre 2009, Revue archéologique de Picardie, $\mathrm{N}^{\circ}$ spécial, 28

DE CleRCQ W., 2011, « Roman rural settlements in Flanders. Perspectives on a "non-villa" landscape in extrema Galliarum », in RoyMANS N., DERKS T. (ÉDS.), Villa Landscape in the Roman North. Economy, culture and lifestyles, Amsterdam Archaeological Studies, 17, Amsterdam, Amsterdam University Press, p. 235-258

Dufour J.-Y., 2007, « Trois bergeries médiévales à Roissy-en-France (Val-d'Oise) », Archéologie médiévale, 37, p. 75-94.

FeChNer K., Devos Y., LeOPOld M, VÖlKel, J. (ÉDS.), 2011a, Archaeology, soil- and life sciences applied to enclosures and fields, Actes de l'European Association of Archaeologists's $12^{\text {th }}$ Annual Meeting, Krakow, 2006, BAR, International Series, 2222.
FECHNER, K. 2011, « Case studies of phosphorus mapping in Late La Tène to Roman enclosures and ponds between Rhine and Seine », in FECHNER K. DeVos Y., LeOPOld M, VÖLKEL, J. (ÉDS.), op. cit., p. 29-64.

Fechner K., De Lil A., Clavel V., Hus J., Teheux E., 2011b, « Méthodes et résultats de la cartographie du phosphore dans des bâtiments allongés du Néolithique dans le nord de la France et en Wallonie », in Bostrn F., Martial E., Praud I. (DIR.), op. cit., p. 275-297.

Hamerow H., 1999, « Anglo-Saxon Timber Buildings: The Continental Connection ", in Sarfatij H. VerWers W.J.H. \& Woltering P.J. (ÉDS.), In discussion with the Past. Archaeological studies presented to W. A. Van Es, The Foundation for Promoting Archaeology, Zwolle, p. 119-128.

Hollevoet Y., 2007, «L'habitat rural du haut Moyen Âge en Flandre maritime », in VERSLYPE, L. (ÉD.) Villes et campagnes en Neustrie. Sociétés -

Économies - Territoires - Christianisation, Actes des XXV Journées Internationales d'Archéologie Mérovingienne de l'A.F.A.M., éd. Monique Mergoil, p. 221-229.

Hulin G., Broes F., Fechner K., à paraître, «Caractérisation de phénomènes anthropiques par la mesure de paramètres magnétiques sur surface décapée. Premiers résultats sur le projet $\mathrm{Canal}$ Seine-Nord Europe », Archéosciences, $\mathrm{n}^{\circ} 36$

Joseph F, Julien M., Leroy-LANGELIN E., LORIN Y., PRAUD I., 2011, « L'architecture domestique des sites du $\amalg^{\mathrm{e}}$ millénaire avant notre ère dans le nord de la France », in Bostyn F., Martial E., Praud I. (DIR.), op. cit., p. 249-273.

LANGOHR R., 2001, « L'anthropisation du paysage pédologique agricole de la Belgique depuis le Néolithique ancien - Apports de l'archéopédologie», Étude et Gestion des Sols, vol. 8, 2, p. 103-118.

ROUPPERT V. Dufour J.-Y, FeCHNER K, 2011, «Confrontation of the archaeological and pedological approaches: $\mathrm{I}^{\mathrm{st}}-\mathrm{IV}^{\mathrm{th}} \mathrm{c}$. AD enclosure with agricultural vocation at the Chapelle SaintNicolas in Saint-Brice-sous-Forêt (Val-d'Oise, France) », in FeChNer K., Devos Y., LeOpold M., VÖLKEL J. (ÉDS.), op. cit., p. 133-142.

Roymans N, 1999, «Man, cattle and the supernatura in the Northwest European plain », in FABECH C. RINGTVED J. (ÉDS.), Settlement and Landscape. Proceedings of a conference in Arhus, Denmark, May 4-7 1998, Jutland Archaeological Society, Copenhagen, p. 291-30o.

SPeK T., 1992, « The age of the Plaggensoils », in Verhoeven A., Vervloet J.A.J. (ÉdS.), The transformation of the European Rural Landscape Methodogical issues and agrarian change 1770-1914, Bruxelles, p. 72-91.

Van Giffen A.E., 1936, « Der Warf in Ezinge, Provinz Groningen und seine Westgermanischen Häuser », Germania, 36, p. 40-47.

VERDIN P., à paraître, «Analyses de phytolithes des zones de stabulation », in DUfOUR J.-Y. (DIR.), Le Village de Roissy, Revue Archéologique d'Île de France.

Waterbolk H.T., 2009, Getimmerd Verleden. Sporen van voor-en vroeghistorische houtbouw op de zand-en kleigronden tussen Eems en IJssel, Groningen, Bakhuis Publishing.

ZiMMERMAN H., 1999, "Why was cattle-stalling introduced in prehistory? The significance of byre and stable and of outwintering ", in FABECH C., RINGTVED J. (ÉDS.), Settlement and Landscape, Actes de la conférence de Arhus, Danemark, 4-7 mai 1998, Copenhagen, Jutland Archaeological Society, p. 301-318. 